\title{
Study of wave packet treatment of neutrino oscillations at Daya Bay
}

\section{Maria Dolgareva* on behalf of the Daya Bay Collaboration}

Joint Institute for Nuclear Researches, Joliot-Curie 6, Dubna, Moscow region, Russia

E-mail: dolgarevaejinr.ru

\begin{abstract}
The wave packet approach to neutrino oscillation resolves contradictions that arise in the standard plane-wave approximation. It also predicts a number of potentially observable effects. This paper discusses the first experimental limits on decoherence effects based on the data of the Daya Bay experiment. The analysis of data collected in 621 days yields an upper limit on the relative wave packet momentum dispersion of $\sigma_{\text {rel }}<0.20$ at $95 \%$ C.L. Furthermore, the effect due to the wave packet nature of neutrino oscillation is found to be insignificant for the Daya Bay experiment, which ensures an unbiased measurement of the oscillation parameters $\sin ^{2} 2 \theta_{13}$ and $\Delta m_{32}^{2}$ within the plane wave model.
\end{abstract}

38th International Conference on High Energy Physics 3-10 August 2016

Chicago, USA

${ }^{*}$ Speaker. 


\section{Introduction}

In the plane-wave approximation each neutrino flavor state is a coherent superposition of mass eigenstates $\left|v_{k}(p)\right\rangle$ with identical momenta $p:\left|v_{\alpha}(p)\right\rangle=\sum_{k=1}^{3} V_{\alpha k}^{*}\left|v_{k}(p)\right\rangle$. This leads to the probability to detect a neutrino originally of flavor $\alpha$ as having flavor $\beta$ :

$$
P_{\alpha \beta}(L)=\sum_{k, j=1}^{3} V_{\alpha k}^{*} V_{\beta j}^{*} V_{\beta k} V_{\alpha j} \mathrm{e}^{-i 2 \pi L / L_{k j}^{\text {osc }}}
$$

Here $L_{k j}^{\text {osc }}=4 \pi p / \Delta m_{k j}^{2}$ is the oscillation length that depends on the squared-mass difference of neutrino mass eigenstates $\Delta m_{k j}^{2}$ and neutrino momentum $p$, and $L$ is a distance between the detector and neutrino source. The plane wave approach is based on assumptions that contradict well-known physics: $[1,2]$

- Mass eigenstates have definite momenta. Thus the distance $L$ cannot be rigorously defined.

- Mass eigenstates are produced and detected coherently, which is not justified.

- Mass eigenstates have identical momenta. This breaks Lorentz invariance.

- An identification $t=L$ assumes $v / c=1$. Tiny corrections due to $v_{k} \neq 1$ lead to a spurious extra factor of two in the oscillation phase.

A self-consistent way to describe neutrino oscillation is based on the use of wave packets [3].

\section{Wave packet approach to neutrino oscillations}

The plane wave eigenstate $\left|v_{k}(p)\right\rangle$ is replaced by a wave packet with a mean momentum $p^{\prime}$ :

$$
\left|\widetilde{v}_{k}\left(p^{\prime}\right)\right\rangle=\int \frac{d p}{2 \pi} f\left(p, p^{\prime}\right)\left|v_{k}(p)\right\rangle,
$$

where $f\left(p, p^{\prime}\right)$ is the neutrino wave function in momentum space and assumed to be Gaussian. The oscillation probability within the wave packet approach [3] depends on the mass splitting $\Delta m_{k j}^{2}$, effective neutrino momentum $p,{ }^{1}$ distance $L$, and relative wave packet momentum dispersion $\sigma_{\text {rel }}=\sigma_{\mathrm{p}} / p$, while $\sigma_{\mathrm{p}}$ is the intrinsic momentum dispersion of the neutrino wave packet: ${ }^{2}$

$$
P_{\alpha \beta}(L)=\sum_{k, j=1}^{3} \frac{V_{\beta k} V_{\alpha k}^{*} V_{\alpha j} V_{\beta j}^{*}}{\sqrt[4]{1+\left(L / L_{k j}^{\mathrm{d}}\right)^{2}}} \exp \left[-\frac{\left(L / L_{k j}^{\mathrm{coh}}\right)^{2}}{1+\left(L / L_{k j}^{\mathrm{d}}\right)^{2}}-D_{k j}^{2}\right] \mathrm{e}^{-i\left(\varphi_{k j}+\varphi_{k j}^{\mathrm{d}}\right)} .
$$

Here $\varphi_{k j}=L / L_{k j}^{\text {osc }}$ is a plane-wave oscillation phase, $\varphi_{k j}^{\mathrm{d}}$ is a correction due to the dispersion of the wave packet:

$$
\varphi_{k j}^{\mathrm{d}}=-\frac{L / L_{k j}^{\mathrm{d}}}{1+\left(L / L_{k j}^{\mathrm{d}}\right)^{2}}\left(\frac{L}{L_{k j}^{\text {coh }}}\right)^{2}+\frac{1}{2} \arctan \frac{L}{L_{k j}^{\mathrm{d}}} .
$$

Three additional quantities control the modified oscillation probability due to the wave packet treatment. They all depend on the $\sigma_{\text {rel }}$ parameter:

$$
L_{k j}^{\mathrm{coh}}=\frac{L_{k j}^{\mathrm{osc}}}{\sqrt{2} \pi \sigma_{\mathrm{rel}}}, \quad L_{k j}^{\mathrm{d}}=\frac{L_{k j}^{\mathrm{coh}}}{2 \sqrt{2} \sigma_{\mathrm{rel}}}, \quad D_{k j}^{2}=\frac{1}{2}\left(\frac{\Delta m_{k j}^{2}}{4 p^{2} \sigma_{\mathrm{rel}}}\right)^{2},
$$

\footnotetext{
${ }^{1}$ Momentum $p$ depends on the mean momenta $p^{\prime}$ of mass eigenstates at the neutrino production and detection [3].

${ }^{2} \sigma_{\mathrm{p}}$ depends on the kinematics of neutrino production and detection in general. In the QM formalism $\sigma_{\text {rel }}=$ const.
} 
where $L_{k j}^{\text {coh }}$ is the coherence length, i.e. the distance at which the interference of neutrino mass eigenstates vanishes due to spatial separation of $\left|v_{k}\right\rangle$ and $\left|v_{j}\right\rangle . L_{k j}^{\mathrm{d}}$ is the dispersion length, the distance at which the wave packet is doubled in its spatial dimension due to the dispersion of waves moving with different velocities. A factor $D_{k j}^{2}$ suppresses the production and detection of massive neutrino states $\left|v_{k}\right\rangle$ and $\left|v_{j}\right\rangle$ in the coherent flavor state.

Thereby the decoherence effects lead to the suppression of the oscillation when $L>L_{k j}^{\text {coh }}$ and $D_{k j}^{2} \gg 1$. Dispersion of the wave packets partially restores the coherence.

\section{Analysis and results}

The data of the Daya Bay reactor antineutrino experiment was studied using the wave packet approach. Daya Bay was designed to measure the neutrino mixing angle $\theta_{13}$. It is composed of eight identically designed detectors in three groups: two detectors in each of two near experimental halls (EH1 and EH2) and four detectors in a far hall (EH3). Antineutrinos from six nuclear reactors with a total thermal power of $17.4 \mathrm{GW}$ are detected via the inverse beta decay reaction in gadolinium-loaded liquid scintillator. The distances between the detectors and reactors are in the range of $0.5-1.9 \mathrm{~km}$. The analysis is based on the data collected in 621 days, corresponding to the statistics of more than $10^{6}$ antineutrino events [4]. Figure 1 displays the ratios of the observed to the expected numbers of IBD events in the absence of oscillation. As seen there, the plane-wave approach fits data well.

As a goodness-of-fit measure the function $\chi^{2}(\eta)=(\mathbf{d}-\mathbf{t}(\eta))^{T} V^{-1}(\mathbf{d}-\mathbf{t}(\eta))$ was used, where $\mathbf{d}$ is the data vector, and $\mathbf{t}(\eta)$ is the corresponding theoretical model vector which depends on the parameters $\eta$. All sources of statistical and systematic uncertainty were considered in the covariance matrix $V$. The marginalized statistic is $\Delta \chi^{2}\left(\eta^{\prime}\right)=\min _{\eta \backslash \eta^{\prime}} \chi^{2}(\eta)-\min _{\eta} \chi^{2}(\eta)$, where $\eta=\left(\sigma_{\text {rel }}, \Delta m_{32}^{2}, \sin ^{2} 2 \theta_{13}, N\right)$ and $N$ is the absolute flux normalization. $\eta^{\prime}=\sigma_{\text {rel }}$ for onedimensional interval and $\eta^{\prime}=\left(\sigma_{\mathrm{rel}}, \Delta m_{32}^{2}\right)$ or $\eta^{\prime}=\left(\sigma_{\mathrm{rel}}, \sin ^{2} 2 \theta_{13}\right)$ for two dimensional regions.

The analysis results are displayed in Figure 2. The top and middle panels contain allowed regions of the parameters $\left(\sigma_{\text {rel }}, \Delta m_{32}^{2}\right)$ and $\left(\sigma_{\text {rel }}, \sin ^{2} 2 \theta_{13}\right)$. The constraints obtained with the fixedlevel $\Delta \chi^{2}$ and Feldman-Cousins methods are in good agreement. The bottom panel shows the one-dimensional $\Delta \chi^{2}$ profile for $\sigma_{\text {rel }}$, yielding the allowed region for $\sigma_{\text {rel }}$ [3]:

$$
2.38 \cdot 10^{-17}<\sigma_{\text {rel }}<0.232 \text { at } 95 \% \text { C.L. }
$$

The values of $\sigma_{\text {rel }}$ can be divided into three intervals. The region of $10^{-16} \ll \sigma_{\text {rel }} \ll 0.1$ is where the impact of wave packet on neutrino oscillation is negligible for the Daya Bay experiment. In the region $\sigma_{\text {rel }} \ll 10^{-16}$ the localization term $D_{k j}^{2}$ suppresses the oscillations. In the region $\sigma_{\text {rel }} \gg 0.1$ the spatial separation $\left(L_{k j}^{\mathrm{coh}}\right)$ and the dispersion $\left(L_{k j}^{\mathrm{d}}\right)$ are important.

The lower limit can be improved further by taking into account the dimensions of the reactor core and the detector: $\sigma_{\mathrm{x}} \lesssim 2 \mathrm{~m}$, where $\sigma_{\mathrm{x}}=1 /\left(2 p \sigma_{\text {rel }}\right)$ is a wave packet spatial width. The constraint is equal to $\sigma_{\text {rel }} \gtrsim 10^{-14}$ for the average momentum $p=4 \mathrm{MeV}$. Such a value of $\sigma_{\text {rel }}$ corresponds to the regime where the localization term can be safely neglected. This allows us to put an upper limit using the modified $\Delta \chi^{2}$ statistics for an one-sided interval:

$$
\sigma_{\text {rel }}<0.20 \text { at } 95 \% \text { C.L. }
$$

The upper limit excludes $\sigma_{\mathrm{x}} \lesssim 10^{-11} \mathrm{~cm}$. 


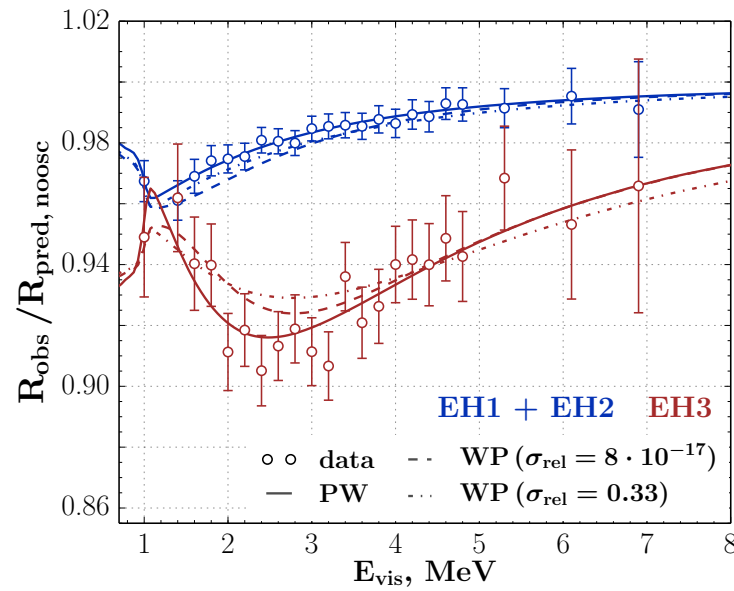

Figure 1: Observed antineutrino spectrum (data points) divided by the spectrum predicted in the absence of oscillations. Red and blue colours correspond to the data from the far (EH3) and near halls (EH1 and EH2) accordingly. Lines show the expected ratios within the plane-wave model (solid line) and wave packet model assuming $\sigma_{\text {rel }}=8 \cdot 10^{-17}$ (dashed line) and $\sigma_{\text {rel }}=0.33$ (dot-dashed line).

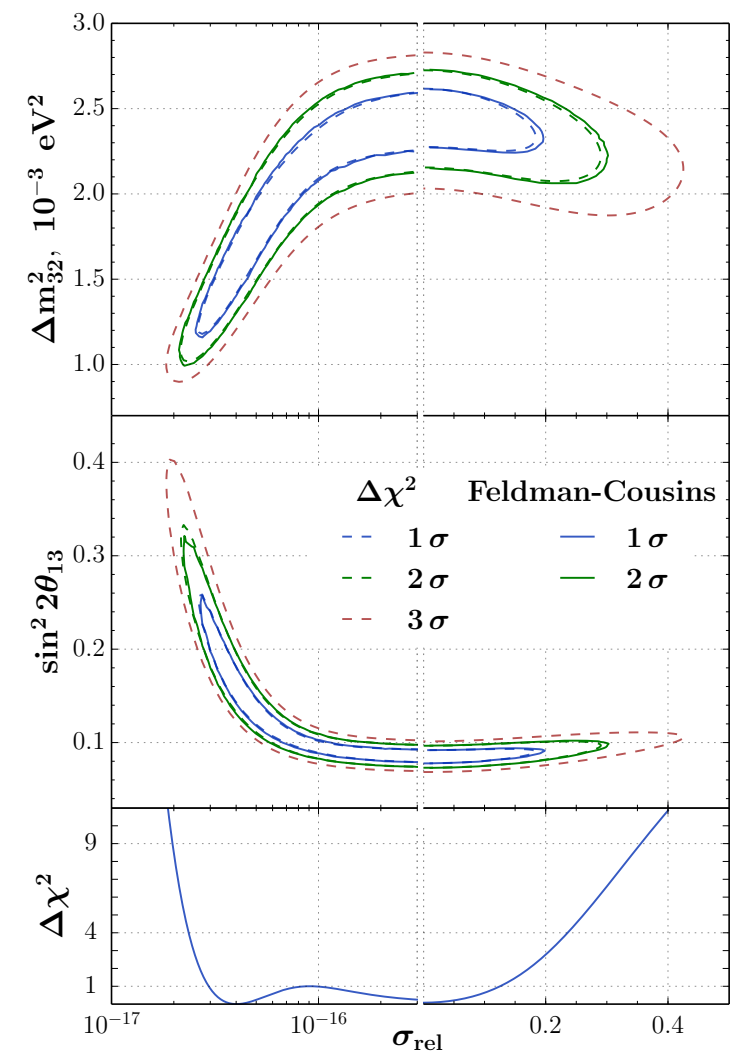

Figure 2: Allowed regions of the parameters $\left(\sigma_{\text {rel }}, \Delta m_{32}^{2}\right)$ (top) and $\left(\sigma_{\text {rel }}, \sin ^{2} 2 \theta_{13}\right)$ (middle) obtained with the fixed-level $\Delta \chi^{2}$ (dashed lines) and the Feldman-Cousins (solid lines) methods. The bottom panel shows the $\Delta \chi^{2}$ profile vs $\sigma_{\text {rel }}$. A break in the middle of the abscissa axis separates the regions of $10^{-17}<\sigma_{\text {rel }}<$ $3 \cdot 10^{-16}$ (logarithmic scale) and $10^{-4}<\sigma_{\text {rel }}<0.5$ (linear scale).

\section{Conclusion}

A first experimental limit on the neutrino wave packet width is obtained: $\sigma_{\mathrm{x}} \geq 10^{-11} \mathrm{~cm}$. The allowed decoherence effect due to the wave packet nature of neutrino oscillation is found to be insignificant for reactor antineutrinos detected by the Daya Bay and other short and medium baseline experiments. This ensures an unbiased measurement of the oscillation parameters $\sin ^{2} 2 \theta_{13}$ and $\Delta m_{32}^{2}$ within the plane wave model.

\section{References}

[1] Evgeny Kh. Akhmedov and Alexei Yu. Smirnov. Paradoxes of neutrino oscillations. Phys.Atom.Nucl. 72 (2009), pp. 1363-1381. arXiv: 0905.1903.

[2] C. Giunti. Coherence and wave packets in neutrino oscillations. Found.Phys.Lett. 17 (2004), pp. 103-124. arXiv: hep-ph/0302026.

[3] Feng Peng An et al. Study of the wave packet treatment of neutrino oscillation at Daya Bay (2016). arXiv: 1608.01661.

[4] F. P. An et al. New Measurement of Antineutrino Oscillation with the Full Detector Configuration at Daya Bay. Phys.Rev.Lett. 115.11 (2015), p. 111802. arXiv: 1505.03456. 